\title{
Experimental Investigations on Tribiological Properties of 6061-T6 Al Alloy by Via Friction Stir Processing
}

\author{
A. Uday Kiran, E. Kavya Pravala \\ Asst.Professor, Dept. of Mechanical Engineering, SVS Group of Institutions,Bheemaram, Warangal Urban,India
}

\begin{abstract}
Microstructure and tribological properties of AlTiB2 nano surface composite fabricated by Friction Stir Processing (FSP) were evaluated. To vary the percentage of TiB2 three different slot thickness viz. $1 \mathrm{~mm}, 1.5 \mathrm{~mm}$ and $2 \mathrm{~mm}$ were considered. Microstructural evaluations showed a nearly uniform distribution of TiB2 in the aluminium matrix after FSP with the addition of composite powder. Microhardness test results shoes FSW of Al6061-T6 alloy with $2 \mathrm{~mm}$ groove width has more hardness. tribological properties were evaluated at two different sliding velocities $0.314 \mathrm{~m} / \mathrm{s}$ and $0.48 \mathrm{~m} / \mathrm{s}$ and results shows that at lower loads there is no much difference in wear rate of surface composite made with different slot sizes but with increase in load and sliding velocity wear rate was increased, however, larger slot Al6061-TiB2 Surface composites show better wear resistance.
\end{abstract}

Keyword-Nano surface composite, TiB2, Friction stir processing, SEM.

\section{INTRODUCTION}

In order to satisfy the industrial desires, standard aluminium needs to get replaced by the composite materials. That to a composite of the same material is preferred to substitute, this is often globally scientists and engineers showing exhaustive interest on the aluminium metal matrix composites. Conventionally these composite were the product of standard technique. The stir casting involves the incorporation of sic and $\mathrm{TiO} 2$ reinforcement particle into the liquid aluminium melt and permits the mixture to solidify. The crucial part of fabrication is to make smart wetting between the particle reinforcement and liquid aluminium alloy melt. The only and most commercially used technique is stir casting or vortex method. The vortex method involves the introduction of preheated reinforcement particles into the vortex of liquefied alloy created by rotating the vane. many aluminium firms adopted this in commercial scale [1].but from both the techniques it is a tedious job to produce a homogeneous composite material and that too, to impart or to enhance the mechanical or tribological properties at a definite space it's not possible with standard fabrication techniques, this may be achieved by friction stir process technique, it's a derived method from friction stir welding.

Friction stir welding (FSW) may be a novel solid-state joining technique that was invented and patented by the welding Institute in 1991. The technique was designed and at first used for butt and lap fastening of ferrous and nonferrous metals and plastics [1]. However, over the last 20 years, the technique has been extensively changed and improved with a major grow that each technology implementation and scientific exploration resulting in commercialization. This has consequently caused to increase in its applications [2] and has evolved to make many new processes, among that is the friction stir process. The method involves clamping the workpieces along on the machine bed on the butting faces of the joint. A non-consumable rotating tool, with a specially designed pin and shoulder, is plunged between the neighbouring faces of the joint and traversed on the joint line. The friction between the tool and the workpiece generates localised heating within the neighbourhood of the immersed rotating pin, inflicting the plastic deformation of the material. Additionally, the generated heat softens the material and creates a plasticized region around the plunged rotating pin, whereas the shoulder of the tool prevents the egress of the plasticized material from the weld. The tool meantime moves linearly on the joint line, inflicting the plasticized material to ensure the front to the rear of the pin, for that purpose, it coalesces and forms a solid-state welded joint [3]. Friction stir welding enjoys various benefits over the previous standard welding techniques. These benefits are classified into two distinct classes that are the metallurgical benefits and the environmental benefits. The metallurgic benefits embrace low distortion, larger weld strength, smart dimensional stability and repeatability, no loss of alloying elements, excellent metallurgical properties within the joint space, smart corrosion resistance, fine microstructure, and absence of cracks [4].

Dharmpal Deepak et al were investigated mechanical properties of surface composite based on 5083Al matrix bolstered with nano-sized carbide particles has been madeup by Friction stir process (FSP). Microstructure, hardness and wear behaviour of the surface composite are investigated 
and compared with those of 5083Al alloy. It's observed that the friction stir processed (FSPed) sample possess higher hardness, particularly within the nugget zone, as compared to 5083Al [5]. E. T. Akinlabi et al have investigated the impact of process parameters on the wear resistance behaviour of friction stir processed Al-TiC composites. This was achieved through microstructural characterization by using both the optical and scanning electron microscope (SEM), Microhardness profiling, and tribological characterization by suggests that of the wear. The Microhardness profiling of the processed samples unconcealed associate enhanced hardness value, that was a function of the tic particles incorporated in comparison to the parent material. The damage resistance property was additionally found to extend as a result of the tic powder addition. The correct combination of process parameters was found to enhance the wear resistance property of the composites produced [6]. Mohammad Narimani et al studied mechanical properties characterization of Al-TiB2 by adding ten percentage of titanium bicarbonate to the aluminium AA6063 Alloy by FSP and discovered that there's a notable increase in tensile strength of surface composites [7]. J. Gandra et al With successive fully overlapped depositions with increasing sic concentration or particle size, a gradient on the coating thickness was produced. Many methods to include sic particles were investigated, as well as, their influence on coating properties, particle distribution and homogeneity [8].

By considering above literature it's been noticed that there's a major improvement in mechanical properties and tribological properties of FSP Surface Composites. It's associated unconventional machining technique to produce the composite material. This lead to current work to analyse the mechanical and tribological properties of surface composites product of aluminium 6061 and titanium bicarbonate by groove filling technique.

\section{MATERIAL AND EXPERIMENTAL METHOD}

The base material used in this study is a 6-mm thick aluminium alloy 6061-T6. The plates were cut into rectangular pieces of $140 \mathrm{~mm} \times 100 \mathrm{~mm}$ dimensions. The chemical composition of the base material is shown in Table 1.

Table I: Chemical composition of aluminium 6061-T6 alloy (wt\%)

\begin{tabular}{|c|c|c|c|c|c|c|c|c|}
\hline Element & $\mathbf{M g}$ & $\mathbf{S i}$ & $\mathbf{C u}$ & $\mathbf{Z n}$ & $\mathbf{T i}$ & $\mathbf{M n}$ & $\mathbf{C r}$ & $\mathbf{A l}$ \\
\hline Amount (wt\%) & 0.85 & 0.68 & 0.22 & 0.07 & 0.05 & 0.32 & 0.06 & Balance \\
\hline
\end{tabular}

The particles TiB2 was supplied by Alfa Aesar, the USA with -325 mesh size. The powder was then milled to nanoscale $(35 \mathrm{~nm})$ using planetary micro mill supplied by FRITSCH, Germany. The particle size of the size of TiB2 is confirmed using particle size analyser. The result is shown in Fig1.

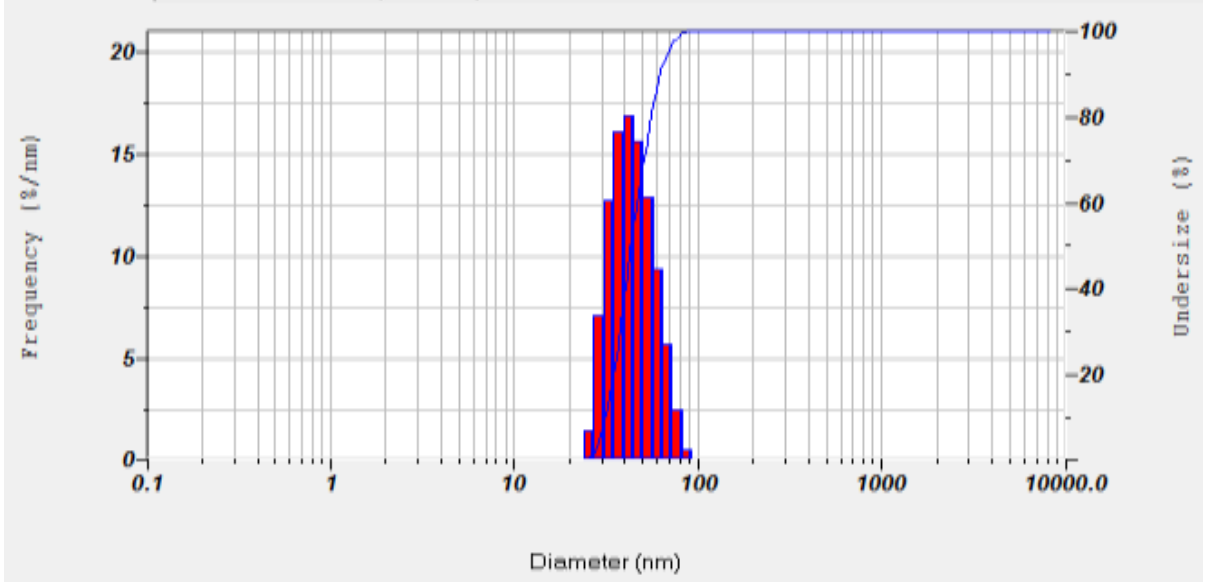

Fig.1: Size of the TiB2 Particles

A square groove was made on the alloy plates with dimensions of $1 \mathrm{~mm}$ width ,2 $\mathrm{mm}$ width and $2.5 \mathrm{~mm}$ depth tangent to the pin on the advancing side, which is $1 \mathrm{~mm}$ far away from the centre line of the tool rotation on the aluminium alloy 6061-T6 plate. The schematic diagram of aluminium alloy plate used for FSP is shown in Fig .2.a. H13 tool steel having screwed taper pin profile with shoulder diameter of $24 \mathrm{~mm}$, pin diameter of $8 \mathrm{~mm}$ and $3.5 \mathrm{~mm}$ height was used which figure which is shown in Fig 2.b. 


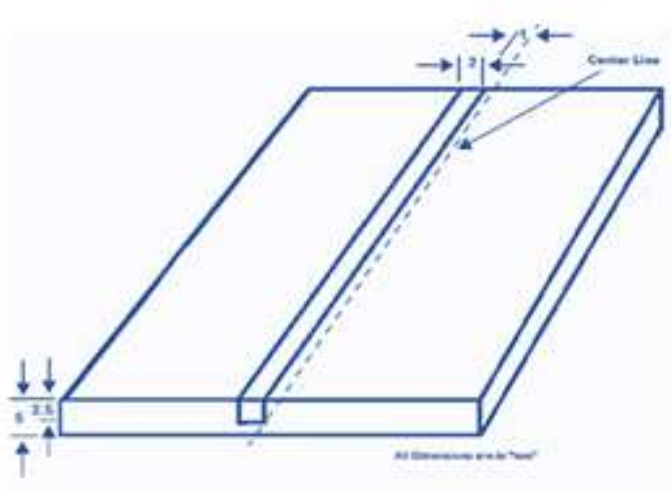

Fig. 2.a. Aluminium alloy plate used for FSP

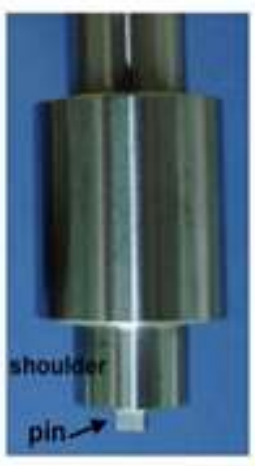

Fig. 2.b. Tool Made of H13 tool steel

$$
\mathrm{k}_{0}=\frac{\mathrm{w}}{\rho \mathrm{LF}}
$$

40rpm Transverse speed with $2^{\circ}$ tilt angle for better mixing.FSPed surfaces were examined by using standard metallographic techniques after etching by Keller's reagent ( $2 \mathrm{ml} \mathrm{HF}, 3 \mathrm{ml} \mathrm{HCl}, 20 \mathrm{ml} \mathrm{HNO}_{3}$ and $175 \mathrm{ml} \mathrm{H}_{2} \mathrm{O}$ ). Both optical microscopy (OM) and scanning electron microscopy (SEM) are used to carry out microstructural observations. The Microhardness of the material was measured on the cross section of specimens and perpendicular to the processing direction using a Vickers digital Microhardness tester (Make: Shimadzu, Model: HMV-2000) with a $50 \mathrm{~g}$ load and duration for $15 \mathrm{~s}$.

\section{A. Abrasive Wear Test}

Abrasive wear studies were carried out under the multipass condition on a pin-on-disc type wear machine supplied by Magnum, Bangalore. Abrasive paper of 200 grade was pasted on a rotating disc (EN 31 Steel disc) of $120 \mathrm{~mm}$ diameter using double-sided adhesive tape. The sample pin was fixed in a holder and was abraded under different applied loads for five intervals of $5 \mathrm{~min}$. where each time interval corresponded to a sliding distance of $94.25 \mathrm{~m}$. The effects of various loads $(10,20,30$ and $40 \mathrm{~N})$ and sliding velocities $(0.314 \mathrm{~m} / \mathrm{s}$ and $0.48 \mathrm{~m} / \mathrm{s})$ in a track radius $50 \mathrm{~mm}$ were studied. The samples were cleaned by using Acetone to remove any debris adhered to sample before and after each run. The weight loss was recorded by weighing the pin to an accuracy of $1 \times 10^{-3} \mathrm{mg}$ using an electronic balance after each run. The specific wear rate $\left(\mathrm{k}_{0}\right)$ was calculated using equation (1)
Where $\mathrm{k}_{0}$ is the specific wear rate in $\mathrm{m}^{3} / \mathrm{Nm}$, w is the weight loss in grammes, $\rho$ is the density of the sample, $\mathrm{L}$ is the sliding distance in meter, and $\mathrm{F}$ is the applied load in Newton. B. Microhardness

The hardness property of the samples was determined using Vickers hardness tester. A diamond indenter in the form of a right pyramid with a square base and an angle $136^{\circ}$ between opposite faces is forced into the material under a load of $5 \mathrm{~N}$. The Vickers hardness number is directly obtained from the test machine and also it can be calculated using the following equation 2 and 3.

$$
\begin{aligned}
& H_{v}=0.1891\left(\frac{P}{d^{2}}\right) \\
& d=\left(\frac{H+V}{2}\right)
\end{aligned}
$$

Where $P$ is the applied load (N), $d$ is the diagonal of square impression (mm), $H$ is the horizontal length (mm) and $V$ is the vertical length (mm).

\section{Scanning Electron Microscopy Analysis}

Scanning electron microscopy (SEM) was used to monitor the fracture surface of the composites after mechanical test of each sample. SEM analysis was performed using Nova Nano SEM 450 at an accelerating voltage of $15 \mathrm{kV}$. The fractured surfaces were gold coated with a thin film to increase the conductance for SEM for analysis.

\section{RESULTS AND DISCUSSION}

A. Microstructure and Microhardness 

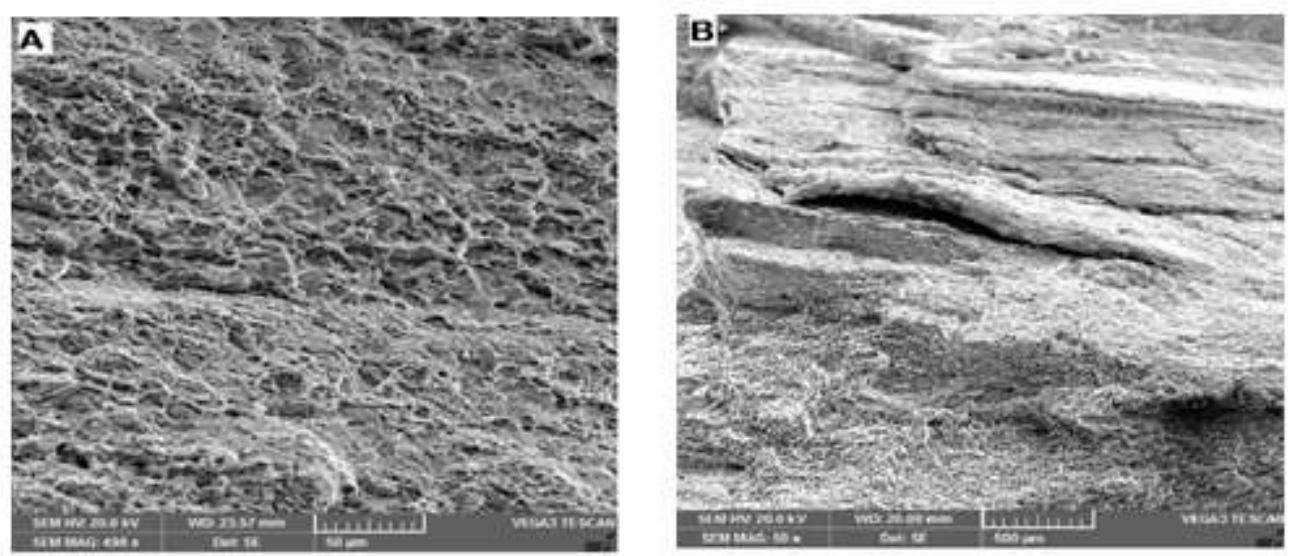

Fig.3: SEM microstructures of Al/TiB2 nano surface composites a) at 498x magnification b) at $50 x$ magnification

Fig. 3.a and 3.b are SEM images of the surface composite fabricated FSP. The black particles are TiB2. As it shown in Figure. 3.a which is observed at $498 \mathrm{x}$ magnification, the distribution of TiB2 particles is very uniform and no obvious microporosity defect can be observed. Furthermore, this figure illustrated the interface condition of TiB2 particles with substrate observed by SEM. Fig 3.b is the same surfac studied at 50x magnification, here it is observed as the mixture as heterogeneous and mere amount if Tib2 particles are stipulated at one point.

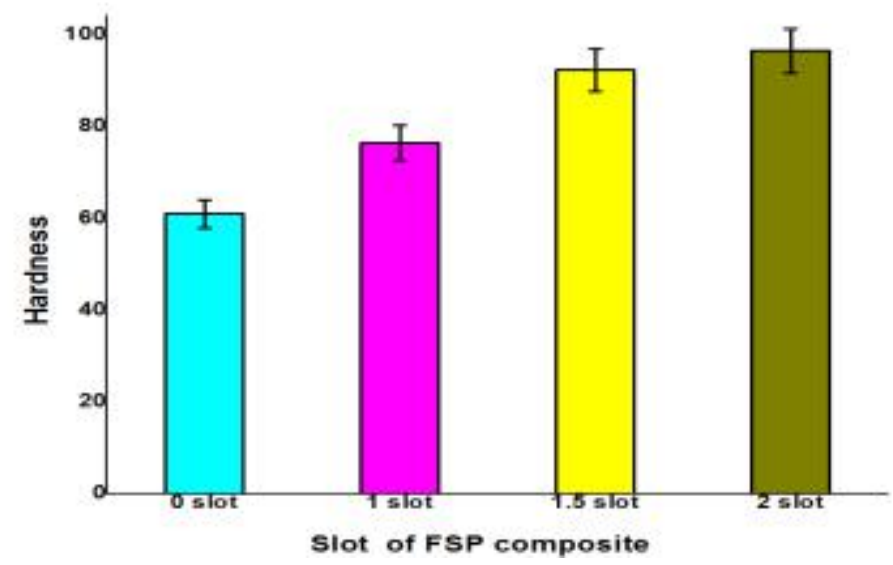

Fig. 4. Vickers Microhardness values

Fig.4 represents the microhardness test result, which is a relation between hardness and width of the slot in which Tib2 is filled for the FSP. 0 slot indicate that pure aluminium and other are respective slot sizes. This is evident that increase in the size of the slot will increase surface hardness up to some extent rapidly after that even though there is an increase in hardness it is not as much as before. In the current study, it is observed that FSP composite with $1 \mathrm{~mm}$ slot thickness shows an increase in hardness by $18 \mathrm{Hv}$, changing the slot thickness $1 \mathrm{~mm}$ to $1.5 \mathrm{~mm}$ hardness increased by $20 \mathrm{Hv}$ and increasing the slot size from $1.5 \mathrm{~mm}$ to $2 \mathrm{~mm}$ increase in hardness is only $3 \mathrm{Hv}$.

\section{B. Tribological properties}

The wear rate with respect to the load on the Al-TiB2 nano surface composites at different sliding velocities $(0.314 \mathrm{~m} / \mathrm{s}$ and $0.48 \mathrm{~m} / \mathrm{s}$ ) is shown in Fig. 5 and Fig.6. It is observed that at both velocities the increasing the slot width of TiB2 particles decreases the wear rate. This is due to the enhanced hardness by the dispersion TiB2 particles and acted as loadbearing elements. 


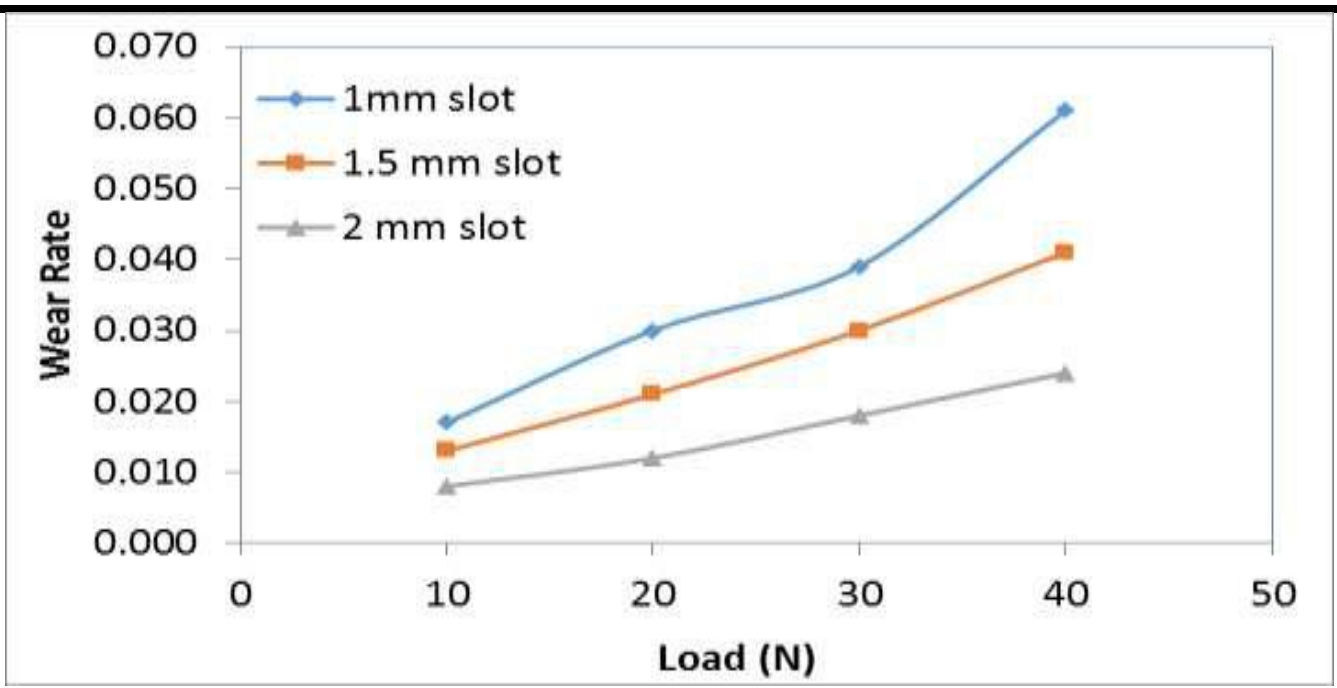

Fig. 5: Wear rate of Al-TiB2 nano surface composites at sliding velocity 0.314

It is observed that the increasing the volume percentage of TiB2 particles decreases the wear rate. It is observed that slot thickness i.e volume of TiB2 particles in the Aluminium doesn't have much impact at lower loads but with the enhancement of the load volume of particles shows much significance. It is also observed that increase in the sliding velocity wear rate was increased for the same material at the same load. The lower wear rate was obtained at the optimum loading condition of $10 \mathrm{~N}$ load and $1 \mathrm{~mm}$ slot width of TiB2.
The wear morphology of Al-TiB2 nano surface composites is shown in Fig.7. It can be seen that the much ploughing has been taken at $1 \mathrm{~mm}$ slot thickness, for the $1.5 \mathrm{~mm}$ slot and $2 \mathrm{~mm}$ slot specimens is observed that less wear and ploughing of surface Due to presence of hard nano TiB2 particles, however base Aluminum alloy surface is subjected to wear where ever absence of TibB2 Particles and formed as rough surface.

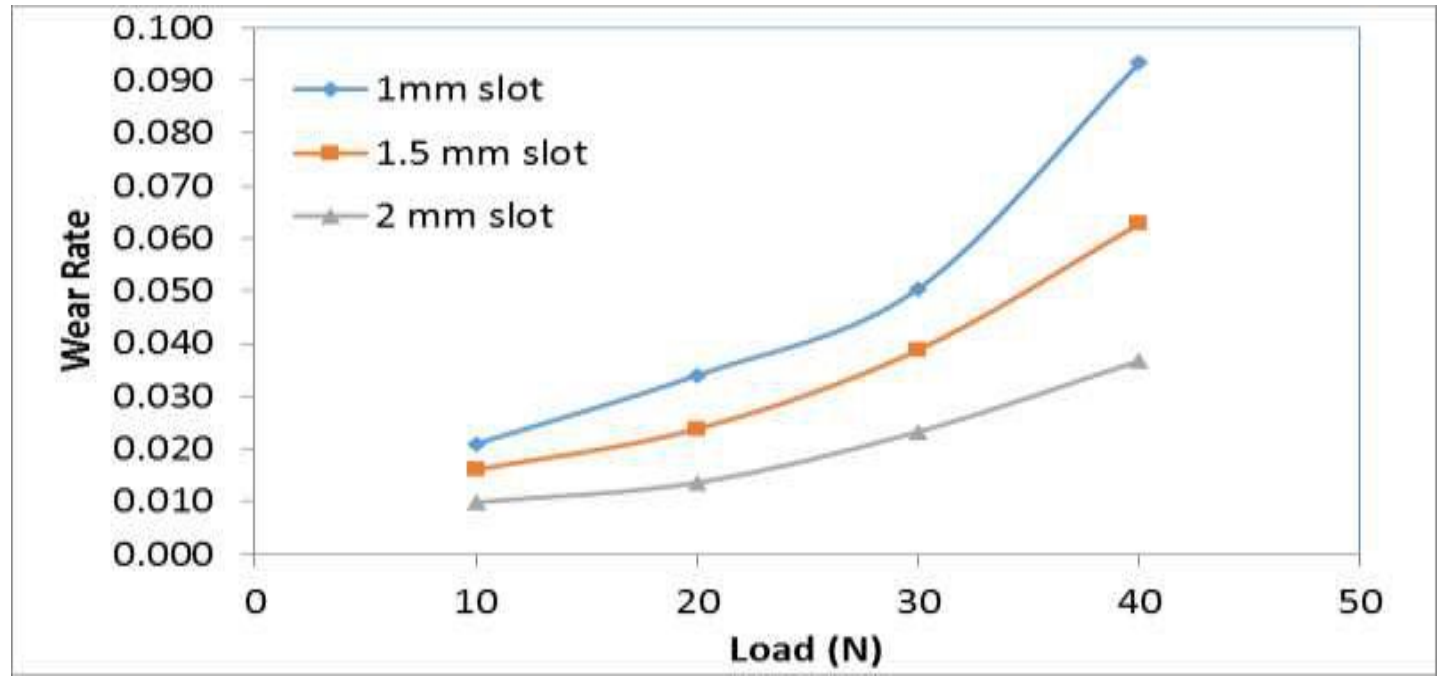

Fig. 6: Wear rate of Al-TiB2 nano surface composites at sliding velocity 0.48 

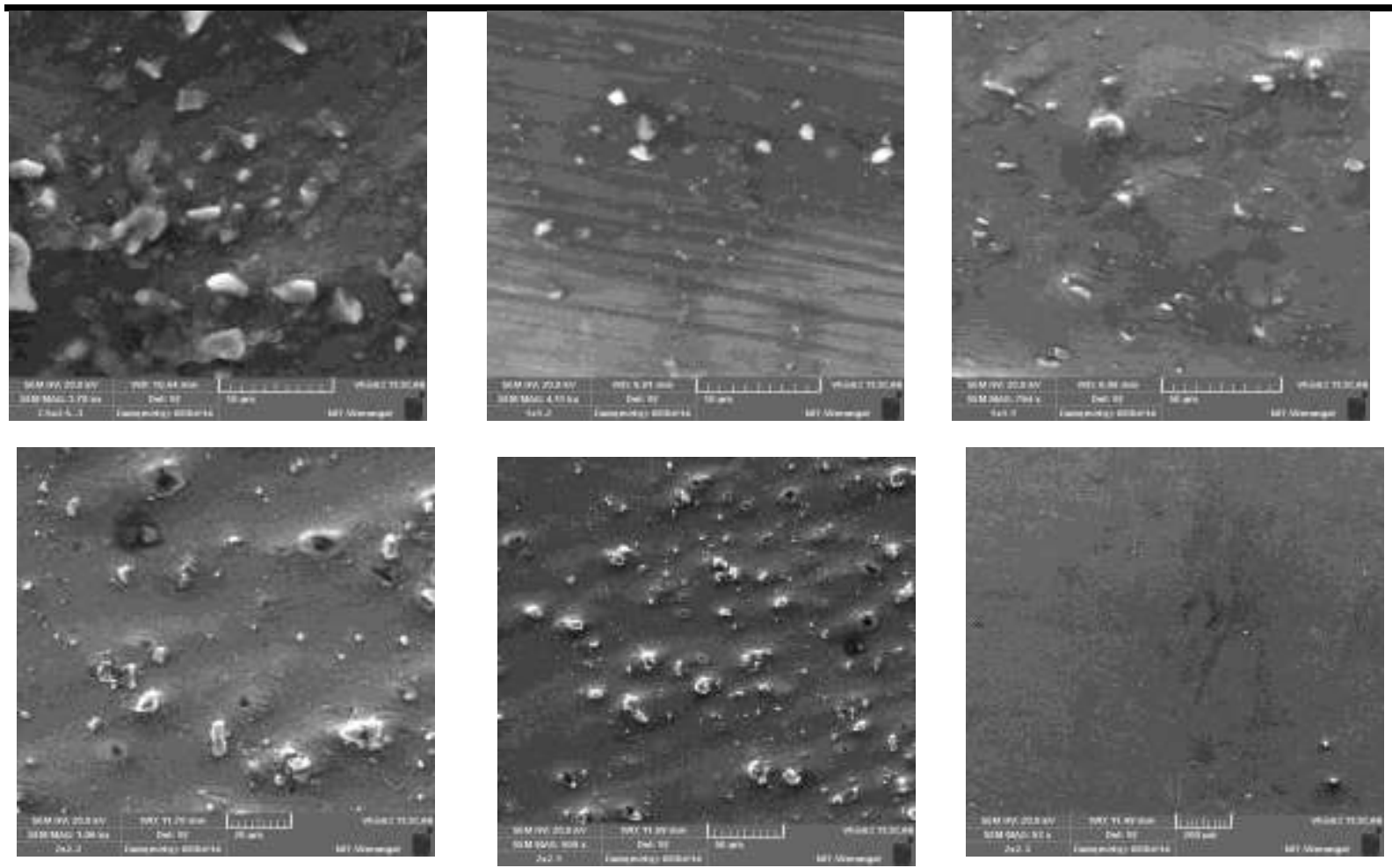

Fig.7: Wear morphology of Al-TiB2 nano surface composites

\section{CONCLUSION}

The nanocomposite surface layer by reinforcing TiB2 particles on Aluminum 6061-T6 Alloy via FSP successfully fabricated. Effect of nano-sized reinforcement particles such as TiB2 (average size is $35 \mathrm{~nm}$ ) on microstructure, Microhardness and Tribological properties of Aluminum 6061-T6 alloy based nano surface composites fabricated via FSP was studied by varying the slot thickness and the following conclusions are to be obtained.

- The increase in the size of the slot will increase surface hardness up to some extent rapidly after that even though there is an increase in hardness it is not as much as before

- It is observed that increase in slot width of TiB2 as $2 \mathrm{~mm}$ Microhardness increases up to $98 \mathrm{Hv}$ and which is higher than as base Aluminium alloy i.e. slot width as $0 \mathrm{~mm}$ ( $60 \mathrm{Hv}$ )

- It is found that slot thickness i.e. volume of TiB2 particles in the Aluminium and sliding velocity doesn't have much impact at lower loads but with the enhancement of the load wear rate is high

\section{REFERENCES}

[1] R. S. Mishra and M. W. Mahoney, "Friction stir processing: a new grain refinement technique to achieve high strain rate superplasticity in commercial alloys," Materials Science Forum, vol. 357, no. 3, pp. 507-512, 2001.
[2] R. S. Mishra and Z. Y. Ma, "Friction stir welding and processing”, Materials Science and Engineering R: Reports,vol.50, no.1-2, pp. 1-78, 2005.

[3] W. M. Thomas and E. D. Nicholas, "Friction stir welding for the transportation industries," Materials and Design,vol.18,no.4-6, pp. 269-273, 1997.

[4] Z. Y. Ma, "Friction stir processing technology: a review," Metallurgical and Materials Transactions A: Physical Metallurgy and Materials Science,vol.39,no.3,pp.642-658,2008

[5] Deepak, Dharmpal, Ripandeep Singh Sidhu, and V. K. Gupta. "Preparation of 5083 Al-SiC surface composite by friction stir processing and its mechanical characterization." International Journal of Mechanical Engineering 3.1 (2013).

[6] Akinlabi, Esther T., et al. "Processing parameters influence on wear resistance behaviour of friction stir processed Al-TiC composites." Advances in Materials Science and Engineering 2014 (2014).

[7] Narimani, Mohammad, Behnam Lotfi, and ZohrehSadeghian. "Investigating the microstructure and mechanical properties of Al-TiB 2 composite fabricated by Friction Stir Processing (FSP)." Materials Science and Engineering: A 673 (2016): 436-442.

[8] Gandra, J., et al. "Wear characterization of functionally graded Al-SiC composite coatings produced by Friction Surfacing." Materials \& Design 52 (2013): 373-383. 